\title{
Global stability analysis of a metapopulation SIS epidemic model* $^{*}$
}

\author{
Abderrahman Iggidr ${ }^{1 \dagger}$, Gauthier Sallet ${ }^{1,2}$, Berge Tsanou ${ }^{1,3}$ \\ ${ }^{1}$ MASAIE project-team, INRIA Grand-Est, \\ and University Paul Verlaine-Metz, LMAM-CNRS,UMR 7122 \\ ISGMP Bat. A, Ile du Saulcy, 57045 Metz Cedex 01, France. \\ ${ }^{2}$ UMI-IRD-209 UMMISCO, University Gaston Berger \\ Saint-Louis, Sénǵal. \\ ${ }^{3}$ Department of Mathematics and Computer Sciences \\ Faculty of Sciences, University of Dschang \\ P.O. Box 67 Dschang, Cameroon \\ and UMI-IRD-209 UMMISCO-Yaoundé, Cameroon. \\ e-mail: Abderrahman.Iggidr@inria.fr \\ Gauthier.Sallet@inria.fr \\ bergetsanou@yahoo.fr
}

October 30, 2011

\begin{abstract}
The conjecture of Arino and van den Driessche (2003) that a SIS type model in a moverstayer epidemic model is globally asymptotically stable is confirmed analytically. If the basic reproduction number $\mathcal{R}_{0} \leq 1$, then the disease free equilibrium is globally asymptotically stable. If $\mathcal{R}_{0}>1$, then there exists a unique endemic equilibrium which is globally asymptotically stable on the nonnegative orthant minus the stable manifold of the disease free equilibrium.
\end{abstract}

Keywords: Metapopulation models; SIS models; nonlinear dynamical systems; global stability; monotone systems.

*A preliminary version has been presented at CARI08, Rabat, 2008.

${ }^{\dagger}$ Corresponding author 


\section{Introduction}

Arino and van den Driessche (2003) considered the so-called "mover-stayer" model adapted from Sattenspiel and Dietz (1995) by adding the age structure of a stationary population. They studied a metapopulation model with full dynamic in each patch in order to describe sexually transmitted diseases. They computed the basic reproduction number $\mathcal{R}_{0}$ which is the average number of new infective generated by a single infective living in a completely susceptible population during his/her entire life span (Diekmann et al., 1990; Diekmann and Heesterbeek, 1999; van den Driessche and Watmough, 2002; Artzrouni, 2009). They also established bounds on $\mathcal{R}_{0}$. We analyze the global stability of the equilibria of this model. We use a theorem of Hirsch (1984) and confirm the conjecture of Arino and van den Driessche (2003).

Arino et al. (2005) studied a multi-species multi-patch SEIR model, using the concept of asymptotic autonomous systems (Castillo-Chavez and Thieme (1995)) to prove the global stability of the disease free equilibrium (DFE). When $\mathcal{R}_{0}>1$, they established the uniqueness and stability of the endemic equilibrium numerically. Arino et al. (2007) introduced quarantine in a SEIRS compartmental model for a multi-species multi-patch disease. Wang and Zhao (2004) in a SIS model with "pseudo mass-action", assuming that the graph of migration is strongly connected, proved that the DFE is globally attractive when $\mathcal{R}_{0}<1$, and that the disease is uniformly persistent when $\mathcal{R}_{0}>1$. Jin and Wang (2005) showed that if $\mathcal{R}_{0}>1$, there exists a unique endemic equilibrium which is globally asymptotically stable when the dispersal rates of susceptible and infective are equal. Auger et al. (2008) considered the Ross-Macdonald malaria model on $n$ patches. They proved that if $\mathcal{R}_{0} \leq 1$, the DFE is globally asymptotically stable (GAS), and when $\mathcal{R}_{0}>1$, the unique endemic equilibrium is also GAS. Iggidr et al. (2010) described the spatial propagation of a disease which confers no immunity. They proved that if $\mathcal{R}_{0} \leq 1$, then the DFE is GAS; if $\mathcal{R}_{0}>1$ there exists a unique GAS endemic equilibrium. Norman 
and Bowers (2007) considered a multi-groups model in order to study the effectiveness of different vaccination strategies.

\section{The migration model}

\section{Notations and definitions:}

Residents of patch $i$ : individuals who were born and reside in patch $i$;

Travelers: individuals who, at the current time, are not in the patch they reside in;

$n$ : the total number of patches;

$N_{i j}$ : the total number of residents of patch $i$ who are present in patch $j$ at time $t$;

$N_{i}^{r}=\sum_{j=1}^{n} N_{i j}$ : the total resident population of patch $i$ at time $t$;

$N_{i}^{p}=\sum_{j=1}^{n} N_{j i}$ : the population of patch $i$ at time $t$ including both residents and travelers;

$g_{i}>0$ : the per head rate at which residents of patch $i$ leave it per time unit;

$m_{i j} \geq 0$ : the proportion of individuals leaving patch $i$ to patch $j$;

$g_{i} m_{j i}:$ the transfer rate from patch $i$ to patch $j$;

$r_{i j} \geq 0:$ the rate at which residents of patch $i$ return to patch $j$;

$d$ : the death rate, equal to the birth rate.

We use the convention that $m_{i i}=0$ and $r_{i i}=0$, so that when $g_{i}>0, \sum_{j=1}^{n} m_{j i}=1$. Individuals do not give birth out of their resident patch and death occurs everywhere.

The migration model is the same as the one considered by Arino and van den Driessche (2003):

$$
N_{i i}^{\prime}(t)=d\left(N_{i}^{r}(t)-N_{i i}(t)\right)+\sum_{j=1}^{n} r_{i j} N_{i j}(t)-g_{i} N_{i i}(t)
$$

and for $j \neq i$

$$
N_{i j}^{\prime}(t)=g_{i} m_{j i} N_{i i}(t)-r_{i j} N_{i j}(t)-d N_{i j}(t)
$$


We assume that some travelers return home. The return matrix $R$ is $R(i, j)=r_{i j}$ and the outgoing matrix $M$ is $M(i, j)=g_{j} m_{j i}$. We assume that these two matrices have the same zeros and nonzeros pattern, because they represent the return to $i$ from $j$ and the outgoing travel from $j$ to $i$ respectively. We assume that these matrices are irreducible, so that the $n$ patches cannot be separated in two isolated groups. It is always possible to reduce the global study to that of irreducible components, thus our assumption does not reduce the generality of our results.

$N_{i}^{r \prime}(t)=0$ means that the population of residents in each patch is constant. However the population size in a patch $i$ can vary.

Theorem 1 (Arino and van den Driessche (2003)) The system given by Eq. (1) and (2), for the initial value $N_{i j}(0)>0$, has the global asymptotically stable equilibrium

$$
\begin{gathered}
\bar{N}_{i i}=\frac{1}{1+g_{i} C_{i}} N_{i}^{r} \\
\bar{N}_{i j}=g_{i} \frac{m_{j i}}{d+r_{i j}} \frac{1}{1+g_{i} C_{i}} N_{i}^{r}
\end{gathered}
$$

where $C_{i}=\sum_{k=1}^{n} \frac{m_{k i}}{d+r_{i k}}$.

The state of the system is given by $n^{2}$ components $N_{i j}$; it is an element of the nonnegative orthant $[0,+\infty)^{n^{2}}$. Define the line-matrix $N$ by:

$$
N=\left(N_{11}, N_{12}, \ldots, N_{1 n}, N_{21}, \ldots, N_{2 n}, \ldots \ldots, N_{n 1}, \ldots, N_{n n}\right)^{T}
$$

The migration model becomes

$$
N^{\prime}(t)=\mathcal{M N}(t)
$$


where $\mathcal{M}=\operatorname{diag}\left(\mathcal{M}_{i i}\right)$ is a block-diagonal matrix, with the block $\mathcal{M}_{i i}$ for all $i$ given by

$$
\mathcal{M}_{i i}=\left(\begin{array}{ccccc}
-g_{i} & r_{i 2}+d & r_{i 3}+d & \ldots & r_{i n}+d \\
g_{i} m_{2 i} & -r_{i 2}-d & 0 & \ldots & 0 \\
g_{i} m_{3 i} & 0 & -r_{i 3}-d & \ldots & 0 \\
\vdots & \vdots & \ddots & \ddots & \vdots \\
g_{i} m_{n i} & 0 & \ldots & 0 & -r_{i n}-d
\end{array}\right)
$$

\section{The complete model}

We assume that the transitions are independent of the disease status, which implies that infectious and susceptible individuals have the same migration rate. With this assumption the demographic change is given by Eq. (6). Let $S_{i j}(t)$ and $I_{i j}(t)$ be respectively the total number of susceptible and infective individuals in patch $i$ who are present in patch $j$ at time $t: N_{i j}(t)=S_{i j}(t)+I_{i j}(t)$ for all $i, j=1, \ldots, n$. We denote by $\beta_{i j k}>0$, the proportion of infectious contacts in patch $j$ between a susceptible of patch $i$ and an infective from patch $k$, and $\kappa_{j}>0$ the average total number of such contacts in patch $j$ per time unit (de Jong et al., 1995; Hethcote, 2000). Subsequently, the infection rate of individuals in patch $j$ is:

$$
\sum_{k=1}^{n} \kappa_{j} \beta_{i k j} S_{i j}(t) \frac{I_{k j}(t)}{N_{j}^{p}(t)} .
$$

The recovery rate $\gamma$ is the same for all patches. The complete epidemic model is then:

$$
\begin{array}{r}
I_{i i}^{\prime}(t)=\sum_{k=1}^{n} r_{i k} I_{i k}(t)-g_{i} I_{i i}(t)+\sum_{k=1}^{n} \kappa_{i} \beta_{i k i} S_{i i}(t) \frac{I_{k i}(t)}{N_{i}^{p}(t)}-(\gamma+d) I_{i i}(t) \\
S_{i i}^{\prime}(t)=\sum_{k=1}^{n} r_{i k} S_{i k}(t)-g_{i} S_{i i}(t)-\sum_{k=1}^{n} \kappa_{i} \beta_{i k i} S_{i i}(t) \frac{I_{k i}(t)}{N_{i}^{p}(t)}+d\left(N_{i}^{r}-S_{i i}(t)\right)+\gamma I_{i i}(t)
\end{array}
$$


and for $j \neq i$,

$$
\begin{array}{r}
I_{i j}^{\prime}(t)=g_{i} m_{j i} I_{i i}(t)-r_{i j} I_{i j}(t)+\sum_{k=1}^{n} \kappa_{j} \beta_{i k j} S_{i j}(t) \frac{I_{k j}(t)}{N_{j}^{p}(t)}-(\gamma+d) I_{i j}(t) \\
S_{i j}^{\prime}(t)=g_{i} m_{j i} S_{i i}(t)-r_{i j} S_{i j}(t)-\sum_{k=1}^{n} \kappa_{j} \beta_{i k j} S_{i j}(t) \frac{I_{k j}(t)}{N_{j}^{p}(t)}-d S_{i j}(t)+\gamma I_{i j}(t) .
\end{array}
$$

As $S_{i j}(t)=N_{i j}(t)-I_{i j}(t)$, it is sufficient to study Eq. (1), (2), (9), and (11). The differential equations governing the total number of infective individuals are:

$$
I_{i i}^{\prime}(t)=\sum_{k=1}^{n} r_{i k} I_{i k}(t)-g_{i} I_{i i}(t)+\sum_{k=1}^{n} \kappa_{i} \beta_{i k i} S_{i i}(t) \frac{I_{k i}(t)}{N_{i}^{p}(t)}-(\gamma+d) I_{i i}(t)
$$

and for $j \neq i$,

$$
I_{i j}^{\prime}(t)=g_{i} m_{j i} I_{i i}(t)-r_{i j} I_{i j}(t)+\sum_{k=1}^{n} \kappa_{j} \beta_{i k j} S_{i j}(t) \frac{I_{k j}(t)}{N_{j}^{p}(t)}-(\gamma+d) I_{i j}(t) .
$$

Define $\mathbf{N}^{p}$ the vector of $\mathbb{R}^{n^{2}}$ given by:

$$
\mathbf{N}^{p}=\left(N_{1}^{p}, N_{2}^{p}, \cdots, N_{n}^{p}, N_{1}^{p}, \cdots, N_{n}^{p}, \cdots \cdots, N_{n}^{p}\right)^{T}
$$

$\mathbf{N}^{p} \gg 0$ means thats all the components of vector $\mathbf{N}^{p}$ are positive. Using the same ordering as that of $N_{i j}$, we define the vector $I$. The system given by Eq. (13) and (14) is written as:

$$
I^{\prime}(t)=\mathcal{D} I(t)-(\gamma+d) I(t)+\operatorname{diag}\left(\mathbf{N}^{p}(t)\right)^{-1} \operatorname{diag}(N(t)-I(t)) B I(t) .
$$


The matrix $\mathcal{D}$ representing migration is a diagonal block matrix $\mathcal{D}=\operatorname{diag}\left(D_{i i}\right)$, where the block diagonal matrices $D_{i i}$ are defined by:

$$
D_{i i}(i, k)=r_{i k}, \quad D_{i i}(k, i)=g_{i} m_{i k}, \quad D_{i i}(k, i)=-g_{i}, \quad D_{i i}(k, k)=-r_{i k} .
$$

namely:

$$
D_{i i}=\left(\begin{array}{ccccc}
-g_{i} & r_{i 2} & r_{13} & \cdots & r_{i n} \\
g_{i} m_{2 i} & -r_{i 2} & 0 & \cdots & 0 \\
g_{i} m_{i 3} & 0 & -r_{i 3} & \cdots & 0 \\
\vdots & \vdots & \ddots & \ddots & \vdots \\
g_{i} m_{n i} & 0 & \cdots & 0 & -r_{i n}
\end{array}\right)
$$

Denoting $e_{i j}$ the canonical basis of $\mathbb{R}^{n^{2}}$, the matrix $B$ is defined by:

$$
B e_{i j}=\sum_{k=1}^{n} \kappa_{j} \beta_{i k j} e_{k j}
$$

$B$ has the form

$$
B=\left(\begin{array}{ccc}
B_{11} & \cdots & B_{1 n} \\
\vdots & \ddots & \vdots \\
B_{n 1} & \cdots & B_{n n}
\end{array}\right) .
$$

The matrix $B$ is the block matrix $B=\left(B_{j k}\right)$, where each block $B_{j k}$ is an $(n \times n)$ diagonal matrix. The $(i, i)$ entry of $B_{k k}$ for every $i$ is equal to $\kappa_{i} \beta_{k k i}$; for $j \neq k$ the $(i, i)$ entry of $B_{j k}$ for every $i$ is $\kappa_{i} \beta_{k j i}$. For every $k$,

$$
B_{k k}=\left(\begin{array}{ccc}
\kappa_{1} \beta_{k k 1} & \cdots & 0 \\
\vdots & \ddots & \vdots \\
0 & \cdots & \kappa_{n} \beta_{k k n}
\end{array}\right)
$$


and for every $(j, k)$, with $j \neq k$,

$$
B_{j k}=\left(\begin{array}{ccc}
\kappa_{1} \beta_{k j 1} & \cdots & 0 \\
\vdots & \ddots & \vdots \\
0 & \cdots & \kappa_{n} \beta_{k j n}
\end{array}\right)
$$

The complete SIS system is given by:

$$
\left\{\begin{aligned}
N^{\prime}(t) & =\mathcal{M} N(t) \\
I^{\prime}(t) & =\mathcal{D} I(t)-(\gamma+d) I(t)+\operatorname{diag}\left(\mathbf{N}^{p}(t)\right)^{-1} \operatorname{diag}(N(t)-I(t)) B I(t) .
\end{aligned}\right.
$$

\section{Properties}

We denote by " $\leq$ " the point-wise ordering in $\mathbb{R}^{n}$, which is the ordering generated by the cone $\mathbb{R}_{+}^{n}$. We also define the classical ordering in $\mathbb{R}_{+}^{n}$ by:

$$
\begin{gathered}
x<y \text { if for any } i x_{i} \leq y_{i} \text { and } x \neq y \\
x \ll y \text { if for any } i x_{i}<y_{i} .
\end{gathered}
$$

\subsection{Positively Invariant Set}

We define the vector:

$$
\mathbf{N}^{r}=\left(N_{1}^{r}, N_{1}^{r}, \cdots, N_{1}^{r}, N_{2}^{r}, \cdots, N_{2}^{r}, \cdots, \cdots, N_{n}^{r}, N_{n}^{r}, \cdots, N_{n}^{r}\right)^{T}
$$

Proposition 1 Define the set

$$
K=\left\{(N, I): 0 \leq N \leq \mathbf{N}^{r} ; 0 \leq I \leq \mathbf{N}^{r}\right\}
$$


Then $K$ is a compact positively invariant set of Eq. (23).

\section{Proof}

We show that the vector field points inward from the faces of $K$.

If $N_{i i}=0$, then

$$
N_{i i}^{\prime}(t)=d N_{i}^{r}+\sum_{j=1}^{n} r_{i j} N_{i j}(t) \geq 0 .
$$

If $\forall j \neq i, N_{i j}=0$, then $N_{i j}^{\prime}(t)=g_{i} m_{j i} N_{i i}(t) \geq 0$. If $N_{i i}=N_{i}^{r}$, then $\forall j, N_{i j}(t)=0$ and $N_{i i}^{\prime}(t)=-d N_{i i}(t)-g_{i} N_{i i}(t) \leq 0$. If $\forall j \neq i, N_{i j}=N_{i}^{r}$, then $N_{i i}=0$ and $N_{i j}^{\prime}(t)=$ $-r_{i j} N_{i j}(t)-d N_{i j}(t) \leq 0$.

If $I_{i i}=0, \forall i$, then

$$
I_{i i}^{\prime}(t)=\sum_{k=1}^{n} r_{i k} I_{i k}(t)+\sum_{k=1}^{n} \kappa_{i} \beta_{i k i} N_{i i}(t) \frac{I_{k i}(t)}{N_{i}^{p}(t)} \geq 0 .
$$

If $\forall j \neq i, I_{i j}=0$, then

$$
I_{i j}^{\prime}(t)=g_{i} m_{j i} I_{i i}(t)+\sum_{k=1}^{n} \kappa_{j} \beta_{i k j} N_{i j}(t) \frac{I_{k j}(t)}{N_{j}^{p}(t)} \geq 0
$$

If $I_{i i}=N_{i}^{r}$, then $I_{i j}(t)=0 \forall j \neq i$, and $I_{i i}^{\prime}(t)=-g_{i} I_{i i}(t)-(\gamma+d) I_{i i}(t) \leq 0$, because $r_{i i}=0$.

If $\forall j \neq i, I_{i j}=N_{i}^{r}$, then $I_{i i}(t)=0, N_{i j}-I_{i j}(t)=N_{i j}(t)-N^{r} \leq 0$, and

$$
I_{i j}^{\prime}(t)=-r_{i j} I_{i j}(t)+\sum_{k=1}^{n} \kappa_{j} \beta_{i k j}\left(N_{i j}(t)-N^{r}\right) \frac{I_{k j}(t)}{N_{j}^{p}(t)}-(\gamma+d) I_{i j}(t) \leq 0 .
$$

Hence the vector field associated with Eq. (1), (2), (13), and (14) points inward from the faces of $K$, so $K$ is positively invariant under the flow of Eq. (23). Moreover, $K$ is a closed bounded subset of $\mathbb{R}^{2 n^{2}}$. 


\subsection{Reduction of Eq. (23)}

Eq. (23) is a triangular system. Theorem 2 reduces the stability analysis to that of a smaller system.

Theorem 2 (Vidyasagar, 1980: Theorem 3.1 and 3.4) Consider the following $\mathcal{C}^{1}$ system

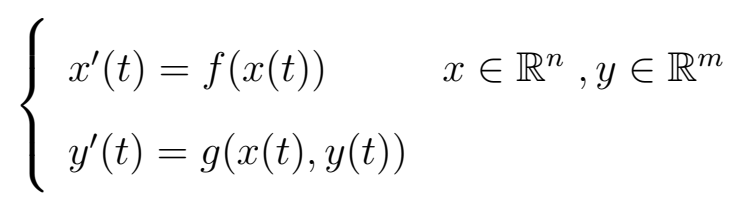

with an equilibrium point $\left(x^{*}, y^{*}\right): f\left(x^{*}\right)=0$ and $g\left(x^{*}, y^{*}\right)=0$.

If $x^{*}$ is globally asymptotically stable $(G A S)$ in $\mathbb{R}^{n}$ for the system $x^{\prime}(t)=f(x(t))$, and if $y^{*}$ is $G A S$ in $\mathbb{R}^{m}$ for the system $y^{\prime}(t)=g\left(x^{*}, y(t)\right)$, then $\left(x^{*}, y^{*}\right)$ is locally asymptotically stable for Eq. (29). Moreover, if all trajectories of Eq. (29) are forward bounded, then $\left(x^{*}, y^{*}\right)$ is GAS for Eq. (29).

We consider Eq. (23) in the positively invariant compact set $K$ and we know that the subsystem $N^{\prime}(t)=\mathcal{M} N(t)$ has a unique globally asymptotically stable equilibrium defined in Theorem 1 . We denote this equilibrium by $\bar{N}$, and the corresponding vector by $\overline{\mathbf{N}}^{p}$. From Theorem 2 it is sufficient to study the stability of the reduced system:

$$
I^{\prime}(t)=\mathcal{D} I(t)-(\gamma+d) I(t)+\operatorname{diag}\left(\overline{\mathbf{N}}^{p}(t)\right)^{-1} \operatorname{diag}(\bar{N}(t)-I(t)) B I(t)
$$

\subsection{Basic reproduction ratio $\mathcal{R}_{0}$}

The basic reproduction ratio was computed by Arino and van den Driessche (2003). Here, we express it in terms of the matrices $B$ and $\mathcal{D}$. We use the framework of Diekmann et al. (1990), Diekmann and Heesterbeek (1999), and van den Driessche and Watmough (2002). We define

$$
\mathcal{F}=\operatorname{diag}\left(\overline{\mathbf{N}}^{p}\right)^{-1} \operatorname{diag}(\bar{N}-I) B I
$$


the function of appearance of new infections in infectious compartments and

$$
\mathcal{V}=\mathcal{D} I-(\gamma+d) I
$$

the transfer in compartments by all other means.

The Jacobian $F$ of $\mathcal{F}$ at the disease free equilibrium is:

$$
F=\operatorname{diag}\left(\overline{\mathbf{N}}^{p}\right)^{-1} \operatorname{diag}(\bar{N}) B
$$

and the Jacobian $V$ of $\mathcal{V}$ is:

$$
V=\mathcal{D}-(\gamma+d) I_{n^{2}}
$$

with $I_{n^{2}}$ the identity matrix of $\mathbb{R}^{n^{2}}$.

The matrix $\mathcal{D}$ is a Metzler matrix (nonnegative off diagonal terms) with a zero column sum. This implies that 0 is a simple eigenvalue of $\mathcal{D}$, the other eigenvalues having a negative real part (Jacquez and Simon, 1993).

The eigenvalues of $V$ are the ones of $\mathcal{D}$ subtracted of $(\gamma+d)$, hence $V$ is a stable Metzler matrix with $-(\gamma+d)$ as stability modulus. This implies that $V$ is nonsingular. We then apply the results of van den Driessche and Watmough (2002) to obtain the basic reproduction ratio:

Proposition 2 The basic reproduction ratio is given by

$$
\mathcal{R}_{0}=\rho\left(-F V^{-1}\right)=\rho\left(-\operatorname{diag}\left(\overline{\mathbf{N}}^{p}\right)^{-1} \operatorname{diag}(\bar{N}) B\left(\mathcal{D}-(\gamma+d) I_{n^{2}}\right)^{-1}\right),
$$

where $\rho(A)$ denotes the spectral radius of the matrix $A$. 


\section{Result}

Theorem 3 We consider Eq. (23) on $K$.

- If $\mathcal{R}_{0} \leq 1$ then the system is globally asymptotically stable at the disease free equilibrium.

- If $\mathcal{R}_{0}>1$ then there exists a unique endemic equilibrium $(\bar{N}, \bar{I})$ with $\bar{I} \gg 0$ which is globally asymptotically stable on $K \backslash\left\{(N, 0), N \in[0,+\infty)^{n^{2}}\right\}$.

\section{Proof}

It is sufficient to study the reduced Eq. (30) which is rewritten as:

$$
I^{\prime}(t)=(F+V) I(t)-\operatorname{diag}\left(\overline{\mathbf{N}}^{p}(t)\right)^{-1} \operatorname{diag}(I(t)) B I(t) .
$$

To prove this theorem, we use Hirsch's (1984) theorem:

Theorem 4 (Hirsch, 1984: Theorem 6.1) Let $F$ be a $C^{1}$ vector field in $\mathbb{R}^{q}$, whose flow $\phi$ preserves $\mathbb{R}_{+}^{q}$ for $t \geq 0$ and is strongly monotone in $\mathbb{R}_{+}^{q}$. Assume that the origin is an equilibrium and that all trajectories in $\mathbb{R}_{+}^{q}$ are bounded. If the matrix-valued map $D F: \mathbb{R}^{q} \rightarrow \mathbb{R}^{q} \times \mathbb{R}^{q}$ is strictly decreasing, in the sense that

$$
\text { if } \quad x<y \text { then } D F(x)>D F(y)
$$

then either all trajectories in $\mathbb{R}_{+}^{q} \backslash\{0\}$ tend to the origin, or there is a unique equilibrium $p,(p \gg 0)$ in the interior of $\mathbb{R}_{+}^{q}$ and all trajectories in $\mathbb{R}_{+}^{q} \backslash\{0\}$ tend to $p$.

The conclusion and the proof of this theorem are similar when $\mathbb{R}_{+}^{q}$ is replaced by a positively invariant subset $K$ of $\mathbb{R}_{+}^{q}$.

Let

$$
X(I)=(F+V) I-\operatorname{diag}\left(\overline{\mathbf{N}}^{p}\right)^{-1} \operatorname{diag}(I) B I
$$


the $\mathcal{C}^{1}$ vector field in $K$.

The flow preserves $K$ for $t \geq 0$. The derivative $D X$ is:

$$
\begin{aligned}
D X(I)= & \mathcal{D}-(\gamma+d) I_{n^{2}}+\operatorname{diag}\left(\overline{\mathbf{N}}^{p}\right)^{-1} \operatorname{diag}(\bar{N}-I) B \\
& -\operatorname{diag}\left(\overline{\mathbf{N}}^{p}\right)^{-1} \operatorname{diag}(B I) .
\end{aligned}
$$

$D X(I)$ being an irreducible $n^{2} \times n^{2}$ Metzler matrix, the flow of $X$ is strongly monotone in $K$. Because each row of $B$ is nonnegative and nonzero, and the matrix-valued map $D X$ is a decreasing function of $I$, so $D X(I)$ is strictly decreasing: if $I_{1}<I_{2}$ then $D X\left(I_{1}\right)>D X\left(I_{2}\right)$. Applying the Theorem 6.1 of Hirsch (1984), we deduce that either all trajectories in $K$ tend to the origin (which is the disease free equilibrium), or there is a unique equilibrium in the interior of $K$ and all trajectories in $K \backslash\left([0,+\infty)^{n^{2}} \times\{0\}\right)$ tend to this equilibrium.

The stability modulus $\alpha(M)$ of a matrix $M$ is the largest real part of the elements of the spectrum $\operatorname{Spec}(M)$ of $M$.

$$
\alpha(M)=\max _{\lambda \in \operatorname{Spec}(M)} \operatorname{Re}(\lambda)
$$

The Jacobian $J(0)$ of Eq. (30) at the disease free equilibrium is $J(0)=F+V . F \geq 0$ and $V$ being a nonsingular Metzler matrix, $F+V$ is a regular splitting of $J(0)$. From Varga (1962), $\rho\left(-F V^{-1}\right)<1$ is equivalent to $\alpha(F+V)<0$. Hence the disease free equilibrium is locally asymptotically stable. Thanks to Hirsch's theorem, the origin is then globally asymptotically stable if $\mathcal{R}_{0}<1$. If $\mathcal{R}_{0}>1$, this is equivalent to $\alpha(J(0))>0$. The disease free equilibrium is then unstable; there exists a unique attracting endemic equilibrium $\bar{I} \gg 0$. This endemic equilibrium $\bar{I}$ satisfies:

$$
(\mathcal{D}-(\gamma+d)) \bar{I}+\operatorname{diag}\left(\overline{\mathbf{N}}^{p}\right)^{-1} \operatorname{diag}(\bar{N}-\bar{I}) B \bar{I}=0
$$


Thanks to the non-negativeness of $B$ and to the fact that $\bar{I} \gg 0$ we get:

$$
D X(\bar{I}) \bar{I}=-\operatorname{diag}\left(\overline{\mathbf{N}}^{p}\right)^{-1} \operatorname{diag}(B \bar{I}) \bar{I}<0 .
$$

Using the fact that $D X(\bar{I})$ is a Metzler matrix, Eq. (42) implies that it is stable (Berman and Plemmons, 1994: criterion $I_{28}$ of Theorem 6.2.3).

The stability modulus then satisfies $\alpha(D X(\bar{I}))<0$. This proves the local asymptotic stability of $\bar{I}$, and using Theorem 4 we deduce that $\bar{I}$ is globally asymptotically stable if $\mathcal{R}_{0}>1$

To complete the proof, we consider the case $\mathcal{R}_{0}=1$, which is equivalent to $\alpha(F+V)=0$. As $F+V$ is an irreducible Metzler matrix, there exists a positive vector $v$ such that $(F+V)^{T} v=0$. We consider the following Lyapunov function

$$
V(I)=\langle I \mid v\rangle
$$

on $K$. The derivative along the trajectories is:

$$
V^{\prime}=-\left\langle\operatorname{diag}\left(\overline{\mathbf{N}}^{p}\right)^{-1} \operatorname{diag}(I) B I \mid v\right\rangle \leq 0 .
$$

This proves the local stability of the disease free equilibrium. By the Theorem 4 of Hirsch we are necessarily in the case where the disease free equilibrium is attractive, which means that the disease free equilibrium is globally asymptotically stable.

\section{Simulations}

We consider two patches with parameters chosen so as to describe gonorrhea (Arino and van den Driessche, 2003). The time unit is a day; the recovery rate is $\gamma=0.04$; 
the average lifespan is 75 years, and $d=1 /(75 \times 365)$. We assume $g_{1}, g_{2}>0$ so that $m_{12}=m_{21}=1$. The disease transmission coefficients are equal in each patch for all contact types: $\beta_{i k j}=\beta_{k}, \forall i, j=1,2$, and

$$
\begin{aligned}
& F=\frac{1}{\bar{N}_{1}^{p} \bar{N}_{2}^{p}}\left(\begin{array}{cccc}
\kappa_{1} \beta_{1} \bar{N}_{11} \bar{N}_{2}^{p} & 0 & \kappa_{1} \beta_{1} \bar{N}_{11} \bar{N}_{2}^{p} & 0 \\
0 & \kappa_{2} \beta_{2} \bar{N}_{12} \bar{N}_{1}^{p} & 0 & \kappa_{2} \beta_{2} \bar{N}_{12} \bar{N}_{1}^{p} \\
\kappa_{1} \beta_{1} \bar{N}_{21} \bar{N}_{2}^{p} & 0 & \kappa_{1} \beta_{1} \bar{N}_{21} \bar{N}_{2}^{p} & 0 \\
0 & \kappa_{2} \beta_{2} \bar{N}_{22} \bar{N}_{1}^{p} & 0 & \kappa_{2} \beta_{2} \bar{N}_{22} \bar{N}_{1}^{p}
\end{array}\right) \\
& =\frac{1}{\bar{N}_{1}^{p} \bar{N}_{2}^{p}}\left(\begin{array}{ll}
\hat{F}_{11} & \hat{F}_{11} \\
\hat{F}_{22} & \hat{F}_{22}
\end{array}\right) . \\
& -V=\left(\begin{array}{cccc}
g_{1}+\gamma+d & -r_{12} & 0 & 0 \\
-g_{1} & r_{12}+\gamma+d & 0 & 0 \\
0 & 0 & r_{21}+\gamma+d & -g_{2} \\
0 & 0 & -r_{21} & g_{2}+\gamma+d
\end{array}\right)=\left(\begin{array}{cc}
V_{11} & 0 \\
0 & V_{22}
\end{array}\right) . \\
& V_{11}^{-1}=\frac{1}{\Delta_{1}}\left(\begin{array}{cc}
r_{12}+\gamma+d & r_{12} \\
g_{1} & g_{1}+\gamma+d
\end{array}\right)=\frac{1}{\Delta_{1}} \hat{V}_{11}^{-1} . \\
& V_{22}^{-1}=\frac{1}{\Delta_{2}}\left(\begin{array}{cc}
g_{2}+\gamma+d & g_{2} \\
r_{21} & r_{21}+\gamma+d
\end{array}\right)=\frac{1}{\Delta_{2}} \hat{V}_{22}^{-1} . \\
& -F V^{-1}=\frac{1}{\Delta_{1} \Delta_{2} \bar{N}_{1}^{p} \bar{N}_{2}^{p}}\left(\begin{array}{cc}
\Delta_{2} \hat{F}_{11} \hat{V}_{11}^{-1} & \Delta_{1} \hat{F}_{11} \hat{V}_{22}^{-1} \\
\Delta_{2} \hat{F}_{22} \hat{V}_{11}^{-1} & \Delta_{1} \hat{F}_{22} \hat{V}_{22}^{-1}
\end{array}\right),
\end{aligned}
$$

where $\Delta_{1}=(\gamma+d)\left(\gamma+d+g_{1}+r_{12}\right)$ and $\Delta_{2}=(\gamma+d)\left(\gamma+d+g_{2}+r_{21}\right)$. 
The matrix of Eq. (6) is a $4 \times 4$ matrix with rank two. Proposition 3 will help us to reduce the computation of its spectral radius to that of a $2 \times 2$ non singular matrix.

Proposition 3 Let $P=\left(\begin{array}{cc}P_{11} & P_{12} \\ P_{21} & P_{22}\end{array}\right)$ be a rank $n$ matrix with $P_{11}, P_{12}, P_{21}, P_{22} n \times n$ square matrices.

If $P_{12}$ is invertible, and $P_{12} P_{21}-P_{12} P_{22} P_{12}^{-1} P_{11}=0$, then

$$
\rho(P)=\rho\left(P_{11}+P_{12} P_{22} P_{12}^{-1}\right)
$$

Moreover, if $P_{12}$ commutes with $P_{22}$,

$$
\rho(P)=\rho\left(P_{11}+P_{22}\right)
$$

\section{Proof}

We use the Schur complement and the properties of the determinant. Recall that, if $A$ is an invertible matrix, and $M=\left(\begin{array}{cc}A & B \\ C & D\end{array}\right)$ is a bloc matrix, then the Schur complement of $A$ in $M$ is the matrix $D-C A^{-1} B$. The Schur complement is usually used to obtain the following factorization: $M=\left(\begin{array}{cc}I_{n} & 0 \\ C A^{-1} & I_{n}\end{array}\right)\left(\begin{array}{cc}A & 0 \\ 0 & D-C A^{-1} B\end{array}\right)\left(\begin{array}{cc}I_{n} & A^{-1} B \\ 0 & I_{n}\end{array}\right)$.

Let $\lambda \in \operatorname{Spec}(P)$, then

$$
\begin{aligned}
\operatorname{det}\left(P-\lambda I_{2 n}\right) & =\operatorname{det}\left(\begin{array}{cc}
P_{11}-\lambda I_{n} & P_{12} \\
P_{21} & P_{22}-\lambda I_{n}
\end{array}\right) \\
& =(-1)^{n} \operatorname{det}\left(\begin{array}{cc}
P_{12} & P_{11}-\lambda I_{n} \\
P_{22}-\lambda I_{n} & P_{21}
\end{array}\right) .
\end{aligned}
$$


$P_{12}$ is invertible and, using the Schur complement $P_{21}-\left(P_{22}-\lambda I_{n}\right) P_{12}^{-1}\left(P_{11}-\lambda I_{n}\right)$ of the last matrix in Eq. (52) yields the factorization

$$
\begin{aligned}
& \left(\begin{array}{cc}
P_{12} & P_{11}-\lambda I_{n} \\
P_{22}-\lambda I_{n} & P_{21}
\end{array}\right)= \\
& \left(\begin{array}{cc}
I_{n} & 0 \\
\left(P_{22}-\lambda I_{n}\right) P_{12}^{-1} & I_{n}
\end{array}\right)\left(\begin{array}{cc}
P_{12} & 0 \\
0 & P_{21}-\left(P_{22}-\lambda I_{n}\right) P_{12}^{-1}\left(P_{11}-\lambda I_{n}\right)
\end{array}\right)\left(\begin{array}{cc}
I_{n} & P_{12}^{-1}\left(P_{11}-\lambda I_{n}\right) \\
0 & I_{n}
\end{array}\right)
\end{aligned}
$$

Then

$$
\begin{aligned}
\operatorname{det}\left(P-\lambda I_{2 n}\right) & =(-1)^{n} \operatorname{det}\left(P_{12}\right) \operatorname{det}\left(P_{21}-\left(P_{22}-\lambda I_{n}\right) P_{12}^{-1}\left(P_{11}-\lambda I_{n}\right)\right) \\
& =(-1)^{n} \operatorname{det}\left(P_{12} P_{21}-P_{12} P_{22} P_{12}^{-1} P_{11}+\lambda\left(P_{11}+P_{12} P_{22} P_{12}^{-1}\right)\right) \\
& =(-\lambda)^{n} \operatorname{det}\left(P_{11}+P_{12} P_{22} P_{12}^{-1}-\lambda I_{n}\right) .
\end{aligned}
$$

The conclusions of Proposition 3 follow.

The application of Proposition 3 to $\left(-F V^{-1}\right)$ yields:

$$
\mathcal{R}_{0}=\frac{1}{\Delta_{1} \Delta_{2} \bar{N}_{1}^{p} \bar{N}_{2}^{p}} \rho\left(\hat{F}_{11}\left(\Delta_{2} \hat{V}_{11}^{-1}+\Delta_{1} \hat{V}_{22}^{-1} \hat{F}_{22} \hat{F}_{11}^{-1}\right)\right) .
$$

Or

$$
\mathcal{R}_{0}=\frac{1}{\Delta_{1} \Delta_{2} \bar{N}_{1}^{p} \bar{N}_{2}^{p}} \rho\left(\Delta_{2} \hat{V}_{11}^{-1} \hat{F}_{11}+\Delta_{1} \hat{V}_{22}^{-1} \hat{F}_{22}\right) .
$$

With Eq. (54), the calculation of $\mathcal{R}_{0}$ is straightforward because we deal with a $2 \times 2$ non singular matrix.

Set

$$
A=\Delta_{2} \hat{V}_{11}^{-1} \hat{F}_{11}+\Delta_{1} \hat{V}_{22}^{-1} \hat{F}_{22}
$$


Then

$$
\mathcal{R}_{0}=\frac{\operatorname{tr}(A)+\left(\operatorname{tr}(A)^{2}-4 \operatorname{det}(A)\right)^{1 / 2}}{2 \Delta_{1} \Delta_{2} \bar{N}_{1}^{p} \bar{N}_{2}^{p}} .
$$

We simulate in order to underline the effect of the migration of individuals on the metapopulation. We compute $\mathcal{R}_{0}$ using Eq. (55). The first parameters are :

$\kappa_{1}=1, \kappa_{2}=1, g_{1}=0.35, g_{2}=0.05, \beta_{1}=0.02, \beta_{2}=0.05, r_{12}=0.2, r_{21}=0.05, N_{1}^{r}=$ $1500, N_{2}^{r}=1500, \gamma=1 / 25, d=1 /(75 \times 365)$.

When the two patches are isolated, the disease will die out in patch 1 with $\mathcal{R}_{0}^{1}=0.5$ while it is endemic in patch 2 with $\mathcal{R}_{0}^{2}=1.25$. When the two patches exchange people according to the parameter values, $\mathcal{R}_{0}=0.96$ : the solutions go to zero and the disease dies out in the metapopulation (Figure 1(a)).

In Figure 1(b), we keep the same parameters as in Figure 1(a) except that $r_{12}$ is decreased significantly from 0.2 to 0.02 . The reproduction number in the metapopulation grows to $\mathcal{R}_{0}=1.1$, whereas $\mathcal{R}_{0}^{1}$ and $\mathcal{R}_{0}^{2}$ remain unchanged because they are not affected by a modification of the mobility coefficients $g_{i}, m_{i j}$, and $r_{i j}$. The disease becomes endemic in the metapopulation. Decreasing $r_{12}$ accounts for lengthening the stay of individuals in patch 2 where the disease is initially endemic. The people from patch 1 spend most of their time in the endemic patch 2 .

The same explanations hold when in Figure 1 (a) $g_{1}$ increases from 0.35 to $0.85\left(\mathcal{R}_{0}=\right.$ 1.03) because $g_{1}$ reflects the flow of individuals from patch 1 to patch 2 . This is shown in Figure 2(c).

Figure 3(a) shows that the metapopulation remains disease free when all patches are initially disease free. In Figure 3(a), we keep the parameters of Figure 1(a) and decrease $\beta_{2}$ from 0.05 to 0.03 .

Figure 3(b) shows that the metapopulation remains endemic when all patches are initially endemic. This is the consequence of our Theorem 3 and of the Theorem 6 of Arino and van den Driessche (2003). In Figure 3(b), we keep the parameters of Figure 1(a) 
and increase $\beta_{1}$ from 0.02 to 0.08 .

\section{Conclusion}

Arino and van den Driessche (2003) showed local stability and studied global behavior numerically. They conjectured the global stability of equilibria depending on the basic reproduction number. We turned this conjecture into Theorem 3.

\section{Acknowledgments}

We thank the anonymous referees for their valuable comments and suggestions.

[Figure 1 about here.]

[Figure 2 about here.]

[Figure 3 about here.]

\section{References}

Arino, J., Davis, J.-R., Hartley, D., Jordan, R., Miller, J.-M., and van den Driessche, P. (2005). A multi-species epidemic model with spatial dynamics. Mathematical Medicine and Biology, 22(2): 129-142.

Arino, J., Jordan, R., and van den Driessche, P. (2007). Quarantine in a multi-species epidemic model with spatial dynamics. Mathematical Biosciences, 206(1): 46-60.

Arino, J. and van den Driessche, P. (2003). A multi-city epidemic model. Mathematical Population Studies, 10(3): 175-193. 
Artzrouni, M. (2009). Transmission Probabilities and Reproduction Numbers for Sexually Transmitted Infections with Variable Infectivity: Application to the Spread of HIV Between Low- and High-Activity Populations. Mathematical Populations Studies, 16(4): 266-287.

Auger, P., Kouokam, E., Sallet, G., Tchuente, M., and Tsanou, B. (2008). The RossMacdonald model in a patchy environment. Mathematical Biosciences, 216(2): 123131.

Berman, A. and Plemmons, R.J. (1994). Nonnegative matrices in the mathematical sciences. Classics in Applied Mathematics. 9. Philadelphia, PA: SIAM.

Castillo-Chavez, C. and Thieme, H. R. (1995). Asymptotically autonomous epidemic models. In Mathematical Population Dynamics : Analysis of heterogeneity, O. Arino, D. Axelrod, M. Kimmel and M. Langlais (eds) Vol. One: Theory of Epidemics. Canada: Wuerz, 33-50

Diekmann, O., Heesterbeek, J.A.P., and Metz, J.A.J. (1990). On the definition and the computation of the basic reproduction ratio $R_{0}$ in models for infectious diseases in heterogeneous populations. Journal of Mathematical Biology, 28(4): 365-382.

Diekmann, O. and Heesterbeek, J. (1999). Mathematical epidemiology of infectious diseases. Model building, analysis and interpretation. Wiley Series in Mathematical and Computational Biology. Chichester: Wiley.

van den Driessche, P. and Watmough, J. (2002). Reproduction numbers and sub-threshold endemic equilibria for compartmental models of disease transmission. Mathematical Biosciences, 180(1-2): 29-48. John A. Jacquez memorial volume.

Hethcote, H. W. (2000). The mathematics of infectious diseases. SIAM Review, 42(4): 599-653. 
Hirsch, M. W. (1984). The dynamical systems approach to differential equations. Bulletin (New Series) of The American Mathematical Society, 11(1): 1-64.

Iggidr, A., Niri, K., and Sallet G. (2010). Analysis of a patchy sis epidemic model. preprint.

Jacquez, J. A. and Simon, C. P. (1993). Qualitative theory of compartmental systems. SIAM Review, 35(1): 43-79.

Jin, Y. and Wang, W. (2005). The effect of population dispersal on the spread of a disease. Journal of Mathematical Analysis and Applications, 308(1): 343-364.

de Jong, M. C., Diekmann, O., and Heesterbeek, H. (1995). How does transmission of infection depend on population size? In Epidemic models. Their structure and relation to data. D. Mollison (ed). Cambridge University Press, 85-94.

Norman, R. and Bowers, R. G. (2007). A Host-Host-Pathogen Model with Vaccination and its Application to Target and Reservoir Hosts. Mathematical Populations Studies, $14(1): 31-56$.

Sattenspiel, L. and Dietz, K. (1995). A structured epidemic model incorporating geographic mobility among regions. Mathematical Biosciences, 128(1-2): 71-91.

Varga, R. (1962). Matrix iterative analysis. (Prentice-Hall Series in Automatic Computation). Englewood Cliffs, New Jersey: Prentice-Hall, Inc. XIII.

Vidyasagar, M. (1980). Decomposition techniques for large-scale systems with nonadditive interactions: Stability and stabilizability. IEEE Transactions on Automatic Control, 25: $773-779$.

Wang, W. and Zhao, X.-Q. (2004). An epidemic model in a patchy environment. Mathematical Biosciences, 190(1): 97-112. 


\section{Infectious individuals}

(a)
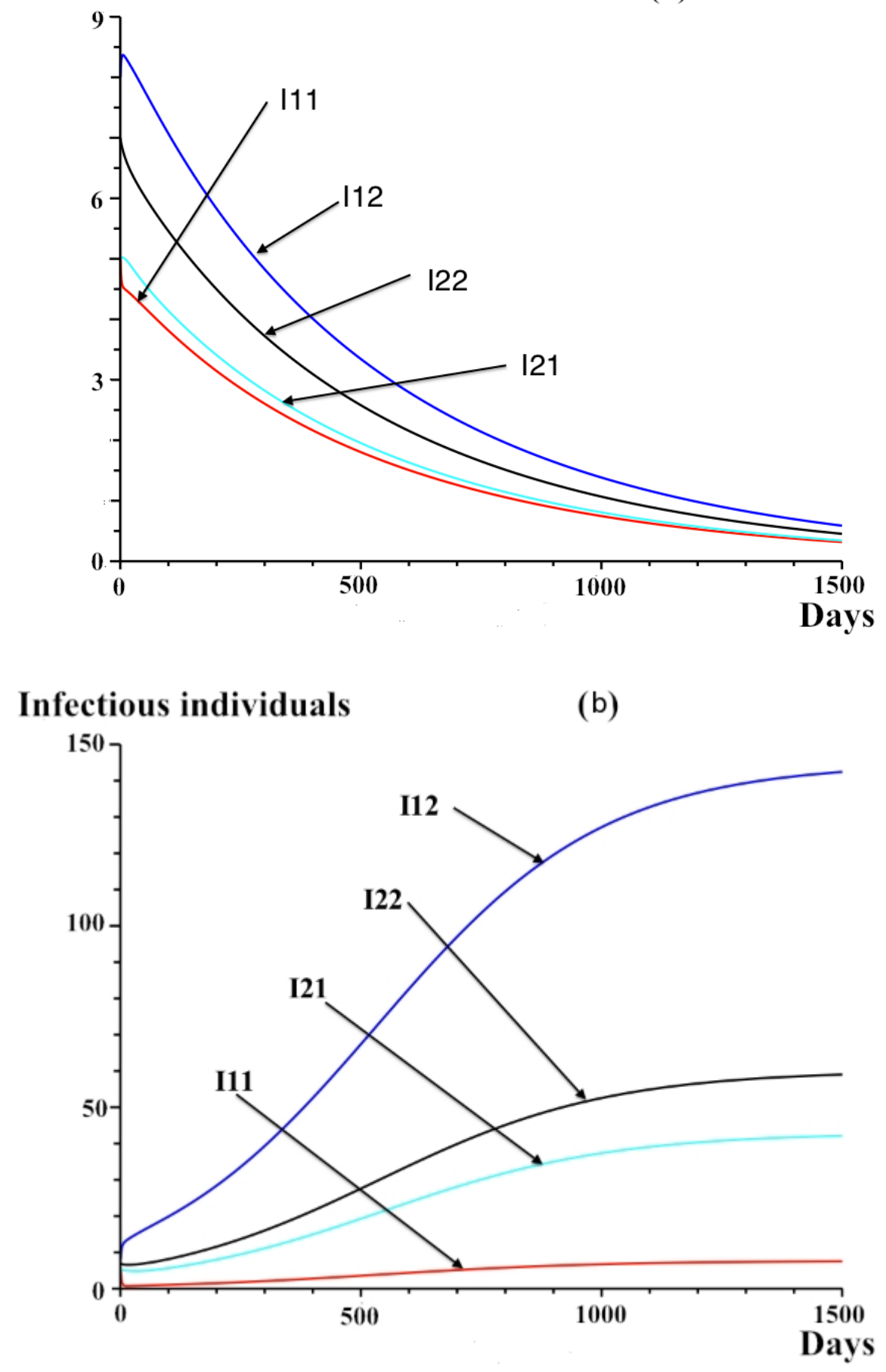

Figure 1: (a) The solutions of the SIS system (23) converge to the DFE when the parameters are (a) $g_{1}=0.35, g_{2}=0.05, \beta_{1}=0.02, \beta_{2}=0.05, r_{12}=0.2: \mathcal{R}_{0}^{1}<1, \mathcal{R}_{0}^{2}>1, \mathcal{R}_{0}<1$. (b) The solutions converge to the endemic equilibrium when the parameters are $g_{1}=0.35, g_{2}=0.05, \beta_{1}=0.02, \beta_{2}=$ $0.05, r_{12}=0.02: \mathcal{R}_{0}^{1}<1, \mathcal{R}_{0}^{2}>1, \mathcal{R}_{0}>1$. 
Infectious individuals

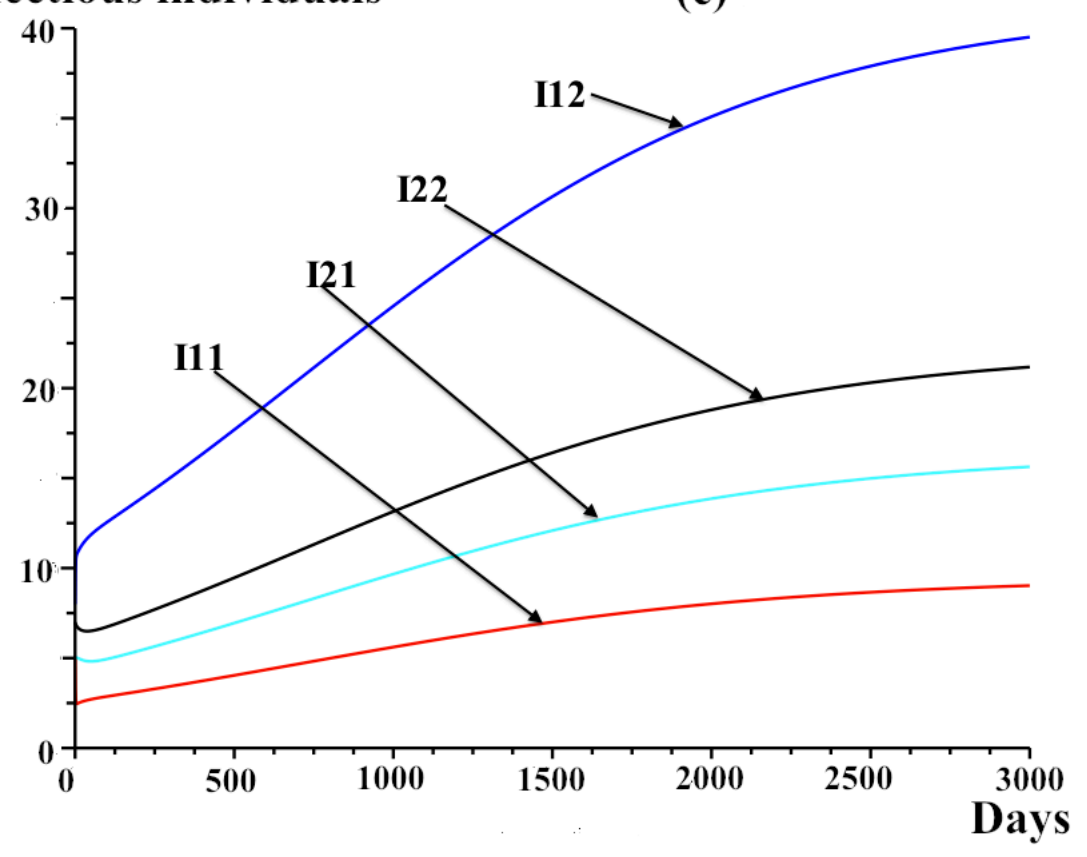

Figure 2: (c) The solutions of the SIS system (23) converge to the endemic equilibrium when the parameters are the same as in Figure 1(a) except $g_{1}=0.85$ instead of $g_{1}=0.35: \mathcal{R}_{0}^{1}<1, \mathcal{R}_{0}^{2}>$ $1, \mathcal{R}_{0}>1$. 
Infectious individuals

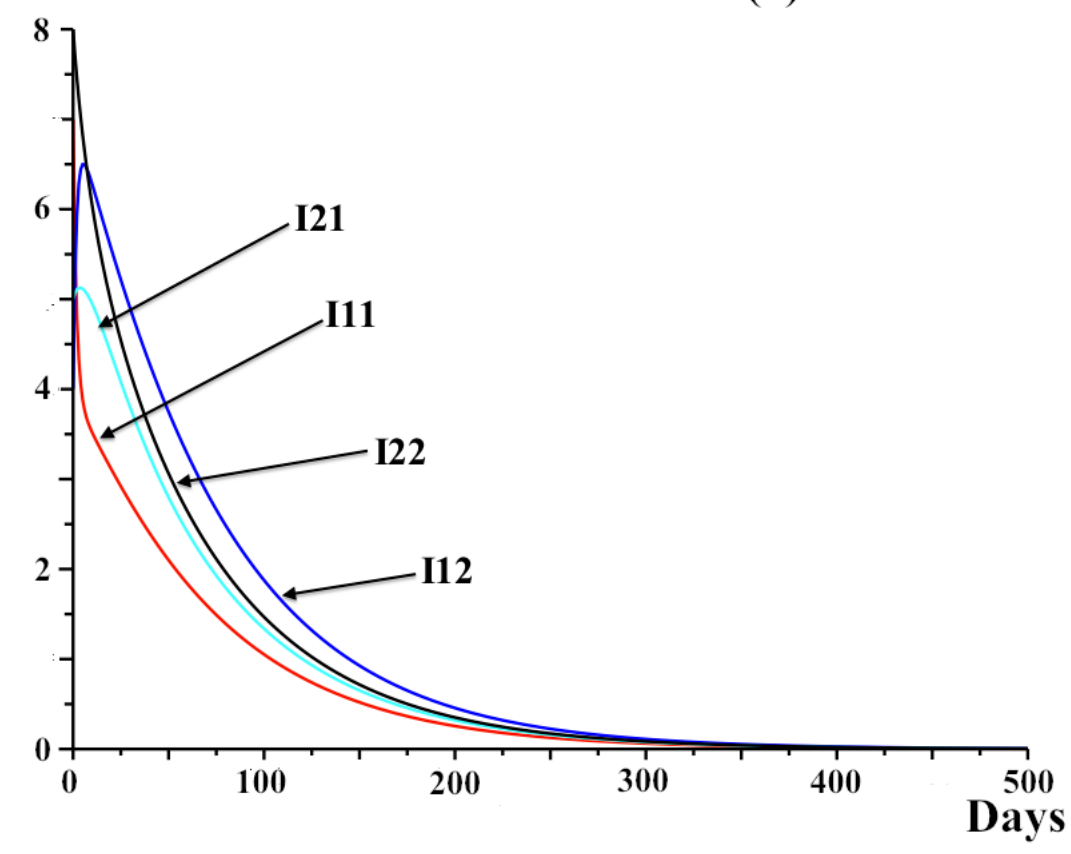

\section{Infectious individuals}

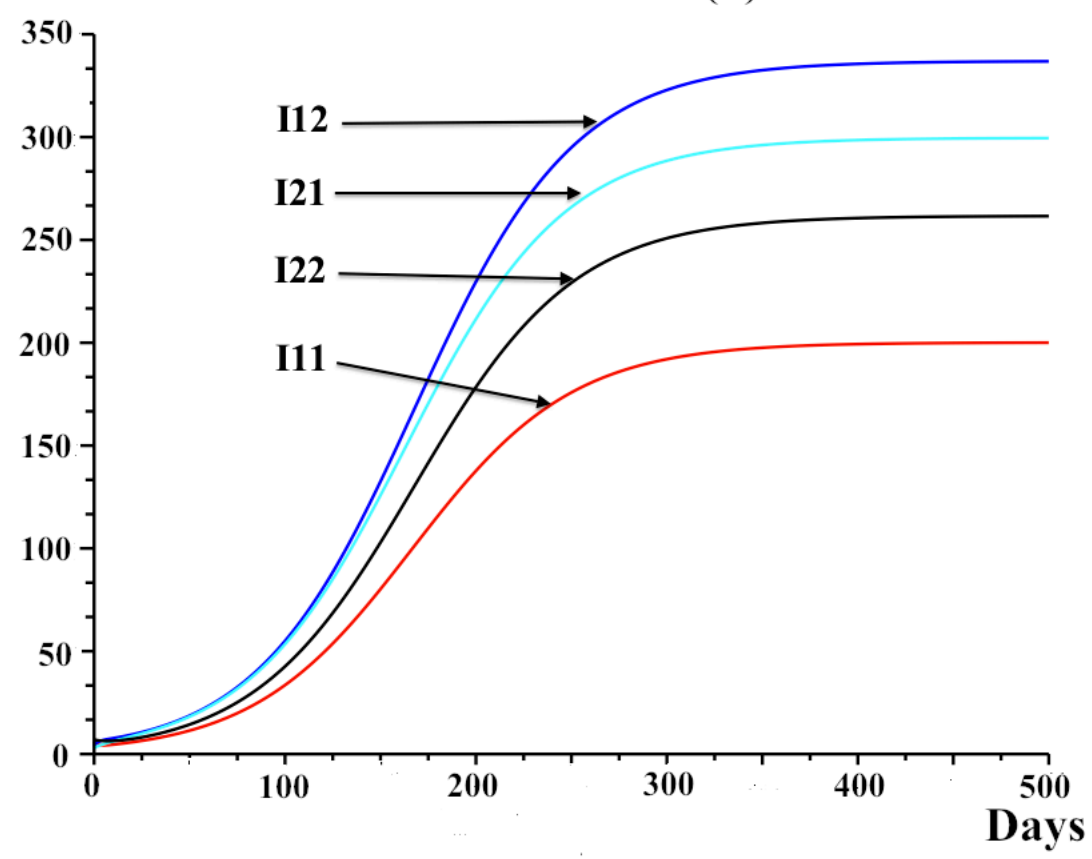

Figure 3: (a) The solutions of the SIS system (23) converge to the DFE when all patches are initially disease free. The parameters are $g_{1}=0.35, g_{2}=0.05, \beta_{1}=0.02, \beta_{2}=0.03, r_{12}=0.2: \mathcal{R}_{0}^{1}<$ $1, \mathcal{R}_{0}^{2}<1, \mathcal{R}_{0}<1$. (b) The solutions of the SIS system (23) converge to the endemic equilibrium when all patches are initially endemic. The parameters are $g_{1}=0.35, g_{2}=0.05, \beta_{1}=0.08, \beta_{2}=$ $0.05, r_{12}=0.2: \mathcal{R}_{0}^{1}>1, \mathcal{R}_{0}^{2}>1, \mathcal{R}_{0}>1$. 